\title{
Nuclei Detection for Drug Discovery using Deep Learning
}

\author{
Nishat Sayyed, Vidit Patil, Mohammed Painter, Deepali Nayak
}

\begin{abstract}
Identifying a cell's nucleus is the starting point for analysis of any kind of drug research. Presently this process is manually carried out by scientists. They take note of each nucleus from microscopic images to begin the drug discovery process. This takes hundreds of thousands of hours for scientific researchers to get their job done. In order to avoid such a bottleneck, this paper proposes an efficient solution using machine learning/ deep learning model. The proposed system can spot nuclei in cell images along with its run-length-encoded code without biologist intervention. A $U$-Net framework is used for the training the model to create efficient system. GPU based system is implemented to get accurate results for storage, retrieval and training of medical cell images. Thus, the system automates the spotting of nuclei thereby drastically reducing time in the drug discovery process.
\end{abstract}

Keywords: microscopy-images, nuclei, segmentation, u-net, deep-learning.

\section{INTRODUCTION}

The traditional drug discovery process by which new medicines can be discovered/identified, requires the biologist to test certain chemicals on a batch of cells to determine if that particular chemical was prone useful or harmful to that batch of cell [1]. As stated by Humayun Irshad et al [1], a nucleus is the most distinguishing feature of a cell, so the biologists need to spot the nuclei in order to observe the change. This process to spot each nucleus in every cell image is time consuming and can increase the time required in the drug discovery process.

The paper is organized in following sections:

Section II gives an overview of the Existing system describing current methods of segmentation and nuclei detection. Section III discusses various challenges in nuclei segmentation and classification. Section IV is the Proposed solution which elaborates on the innovative approach and

methodology to the problem of nuclei detection. Section V is

Revised Manuscript Received on August 19, 2019.

Nishat Sayyed, Final Year Students, Department of Information Technology, Vidyalankar Institute of Technology, Wadala (East), Mumbai 400037,University of Mumbai, Maharastra India. (email :nishatsayyed26@gmail.com)

Vidit Patil,, Final Year Students, Department of Information Technology, Vidyalankar Institute of Technology, Wadala (East), Mumbai 400037,University of Mumbai, Maharastra India. (email:viditpatil14@gmail.com)

Mohammed Painter, Final Year Students, Department of Information Technology, Vidyalankar Institute of Technology, Wadala (East), Mumbai 400037,University of Mumbai, Maharastra, India. (email: mohammedp5253@gmail.com)

Prof. Deepali Nayak, Final Year Students, Department of Information Technology, Vidyalankar Institute of Technology, Wadala (East), Mumbai 400037,University of Mumbai, Maharastra India. (email: deepali.nayak@vit.edu.in) the Implementation section where detailed step-by-step process is elaborated. Section VI is dedicated to the Results of the research experiment. Section VII and Section VIII are Conclusion and Future scope respectively.

\section{EXISTING SYSTEM}

There are many computational algorithms that can find a nucleus and then they can measure the diseased state of cell. However, they work best when nuclei are fairly rounded and not too crowded. Sometimes nuclei can have unusual shapes, or it may be surrounded with tissues as shown in Fig 1. In experiments like this, classical image processing algorithms tend to provide poor results [1], [2].

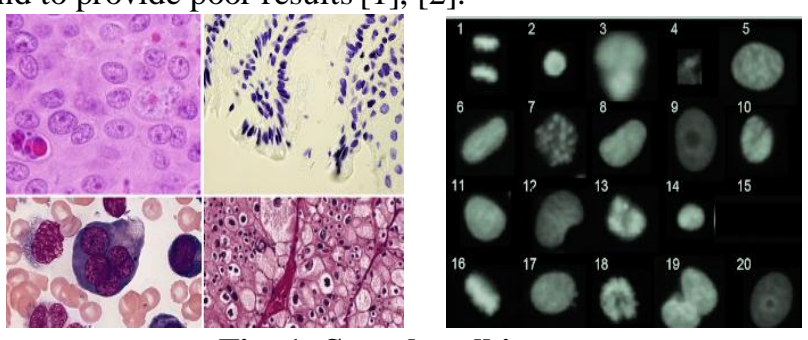

Fig. 1. Sample cell images

Sometimes the biologist has no choice, but to look at every image by eye in order to complete their experiment. This task becomes challenging and time consuming as the number of cell images grow in thousands. Thus there is a need of a system which automatically detects nuclei accurately and reduces overall time required in drug discovery process.

Table I shows an analysis of existing systems comprising of the list of algorithms, methodology used for detecting and segmenting nuclei from cell images and understanding their limitations.

Mask RCNN and Fast RCNN uses instance segmentation as their core methodology and DenseNet and ANN uses semantic segmentation.

1. Semantic segmentation: In this methodology, the system is only context aware i.e. it can only categorize if a particular pixel belongs to one or more classes defined [7], [8], [9].

2. Instance segmentation: In this methodology, the system not only knows about the context (class that the pixel belongs), but also knows the instance at which pixel is

3. present i.e. it is aware about contextual information about an instance [3], [4], [5], [6] 
TABLE I. Analysis of Existing System

TABLE II.

\begin{tabular}{|l|l|l|}
\hline Algorithm & $\begin{array}{l}\text { Methodolog } \\
\boldsymbol{y}\end{array}$ & Limitations \\
\hline Mask RCNN [3] & $\begin{array}{l}\text { Instance } \\
\text { segmentatio } \\
\mathrm{n}\end{array}$ & $\begin{array}{l}\text { Complex and large } \\
\text { number of hidden } \\
\text { layers }\end{array}$ \\
\hline $\begin{array}{l}\text { Fast RCNN [4], } \\
\text { [5] }\end{array}$ & $\begin{array}{l}\text { Instance } \\
\text { segmentatio } \\
\mathrm{n}\end{array}$ & $\begin{array}{l}\text { Requires pretrained } \\
\text { network weights }\end{array}$ \\
\hline DenseNet [6], [7] & $\begin{array}{l}\text { Semantic } \\
\text { segmentatio } \\
\mathrm{n}\end{array}$ & $\begin{array}{l}\text { Static learning rate } \\
\text { throughout the } \\
\text { learning process }\end{array}$ \\
\hline ANN [8] & $\begin{array}{l}\text { Semantic } \\
\text { segmentatio } \\
\mathrm{n}\end{array}$ & $\begin{array}{l}\text { Not very efficient } \\
\text { with image data }\end{array}$ \\
\hline 3D CNN [9] & $\begin{array}{l}\text { Candidate } \\
\text { generation }\end{array}$ & $\begin{array}{l}\text { Need of synthetic } \\
\text { volumes of data }\end{array}$ \\
\hline
\end{tabular}

\section{CHALLENGES IN NUCLEI SEGMENTATION} AND CLASSIFICATION

Table II depicts some of the challenges that are currently faced in nuclei segmentation and classification in varied domains such as Image processing, Data Mining and Cloud Computin

TABLE III. Challenges In Nuclei Segmentation

\begin{tabular}{|c|c|c|}
\hline Domain & Challenges & Description \\
\hline \multirow{3}{*}{$\begin{array}{l}\text { Image } \\
\text { Processing }\end{array}$} & $\begin{array}{l}\text { 1. Crowded } \\
\text { cell images }\end{array}$ & $\begin{array}{l}\text { Cell images with crowded nuclei } \\
\text { (where nuclei are close to each } \\
\text { other) can decrease the efficiency } \\
\text { of fimal results as two nuclei can } \\
\text { be treated as a single nucleus }\end{array}$ \\
\hline & $\begin{array}{l}\text { 2. Nucleus } \\
\text { with } \\
\text { different } \\
\text { shapes }\end{array}$ & $\begin{array}{l}\text { Some nuclei can be fairly round } \\
\text { while others can have unusual } \\
\text { shapes. Due to this, the leamable } \\
\text { parameters can be miscalculated }\end{array}$ \\
\hline & $\begin{array}{l}3 . \quad \text { Cell } \\
\text { image with } \\
\text { more tissue } \\
\text { and muscle } \\
\text { area }\end{array}$ & $\begin{array}{l}\text { Image processing algorithms can } \\
\text { treat muscle areas or tissues as } \\
\text { leamable parameters which'll lead } \\
\text { to false results }\end{array}$ \\
\hline \multirow[t]{2}{*}{ Data mining } & $\begin{array}{l}\text { 1. Working } \\
\text { with image } \\
\text { data }\end{array}$ & $\begin{array}{l}\text { Image formats as input can cause } \\
\text { unnecessary failures due to } \\
\text { incorrect reading or parsing of } \\
\text { image data. }\end{array}$ \\
\hline & $\begin{array}{l}\text { 2. Image } \\
\text { quality }\end{array}$ & $\begin{array}{l}\text { Cell images must be of high } \\
\text { quality which will require minimal } \\
\text { preprocessing }\end{array}$ \\
\hline $\begin{array}{l}\text { Cloud } \\
\text { Computing }\end{array}$ & $\begin{array}{l}\text { 1. Compute } \\
\text { power }\end{array}$ & $\begin{array}{l}\text { Deep leaming requires high end } \\
\text { GPU based systems to train a } \\
\text { model. This training time is more } \\
\text { with image data. Due to this some } \\
\text { models can take days or weeks } \\
\text { just to train the model completely }\end{array}$ \\
\hline
\end{tabular}

\section{LITERATURE SURVEY}

Table III gives a glimpse of all papers reviewed for gaining deeper insights into concepts such as working of CNN, nuclei segmentation including detection of noise etc.
TABLE III .Overview of Literature Surveyed

\begin{tabular}{|c|c|c|}
\hline Papers Reviewed & Architecture & Features \\
\hline Mask R-CNN [3] & Mask R-CNN & $\begin{array}{l}\text { Instance } \\
\text { Segmentation }\end{array}$ \\
\hline $\begin{array}{l}\text { The One Hundred Layers } \\
\text { Tiramisu: } \\
\text { Fully Convolutional } \\
\text { DenseNets for Semantic } \\
\text { Segmentation [8] }\end{array}$ & \begin{tabular}{|l|} 
Fully \\
Convolutional \\
DenseNet
\end{tabular} & $\begin{array}{l}\text { Build very deep } \\
\text { FC-DenseNets } \\
\text { without a feature } \\
\text { map explosion }\end{array}$ \\
\hline \multirow{2}{*}{$\begin{array}{l}\text { Nuclei Segmentation of } \\
\text { Fluorescence Microscopy } \\
\text { Images Using Three } \\
\text { Dimensional } \\
\text { Convolutional Neural } \\
\text { Networks [10] }\end{array}$} & \multirow[t]{2}{*}{ 3D CNN } & $\begin{array}{l}\text { 1. Nucleus } \\
\text { Candidate } \\
\text { Generation }\end{array}$ \\
\hline & & $\begin{array}{l}\text { 2. Overlapping } \\
\text { Nuclei Removal } \\
\text { 3. Blur and Noise }\end{array}$ \\
\hline \multirow[t]{3}{*}{ Fast R-CNN [4] } & \multirow[t]{3}{*}{ Fast R-CNN } & $\begin{array}{l}\text { 1. Higher detection } \\
\text { quality (mAP) than } \\
\text { R-CNN, SPPnet }\end{array}$ \\
\hline & & $\begin{array}{l}\text { 2. Training can } \\
\text { update all network } \\
\text { layers }\end{array}$ \\
\hline & & $\begin{array}{l}\text { 3. No disk storage } \\
\text { is required for } \\
\text { feature caching }\end{array}$ \\
\hline
\end{tabular}

\section{PROPOSED SOLUTION}

This research paper proposes a deep-learning based system that can classify nuclei regions in a cell image. Training the system with thousands of cell images and their nuclei masks helps in building a deep-learning model that predicts the nuclei mask on an image. The system is trained using the U-Net [12] model.

One of the famous Fully Convolutional Networks (FCN) in biomedical image segmentation is U-Net. This approach is suitable for the proposed solution. Because unlike a typical

$\mathrm{CNN}$, it up-samples the classified image to its original resolution using transposed-convolutions. This not only segments the image but also retains the original cell image quality.

The aim is to build an efficient machine learning/ deep learning model that can spot nuclei in cell images with no biologist intervention.

It will save researchers hundreds of thousands of hours each year. No more configuring image processing algorithms and certainly no more looking with the eye. The motive is to build a model that can predict nuclei mask of any cell image regardless of the type of stain used, the species of the cell type, the microscope, the resolution and so on. 


\section{IMPLEMENTATION}

Fig 2 shows a high-level overview of the system. It gives an idea about the system input, different processes involved in the system and finally the prediction is displayed to the users. In the following sub-sections each component is discussed in detail.

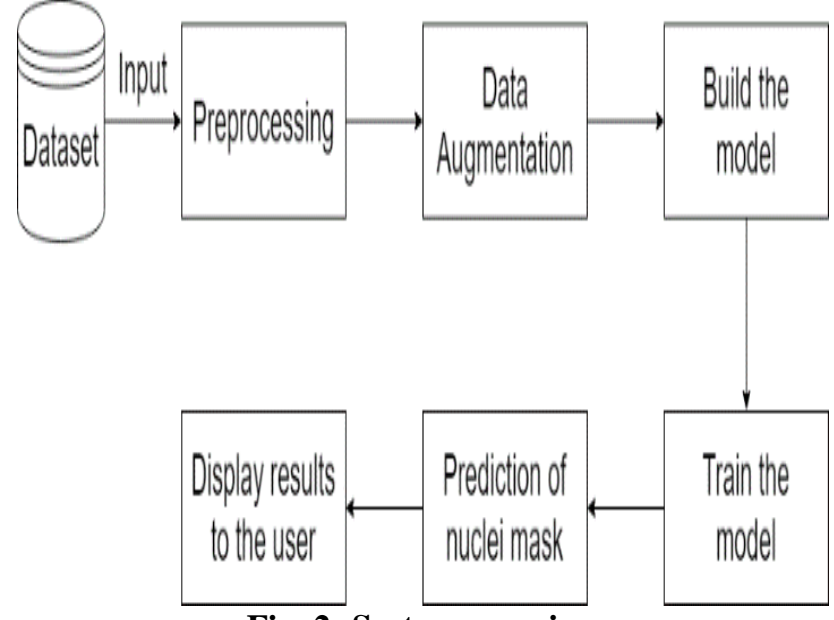

Fig. 2. System overview

\section{A. Dataset}

The dataset is from Kaggle [23] that has a number of cell images with their corresponding annotated mask images. The dataset has training data and test data separately. In the training set there are cell images with annotated mask images and the test set only has the cell images.

The training set has captured cell images and more than one nucleus mask for each image. For example, if an image consists of four nuclei at different positions then there will be four mask images each showing the segmented region and position of the nucleus in the cell image.

The images in the dataset are of different categories that covers a large range of varieties of cell images like different resolution, stain-type, microscope etc. The dataset is an aggregate of different set of histopathology images.

\section{B. Preprocessing}

Fig 3 diagrammatically represents the preprocessing activities performed by the system.

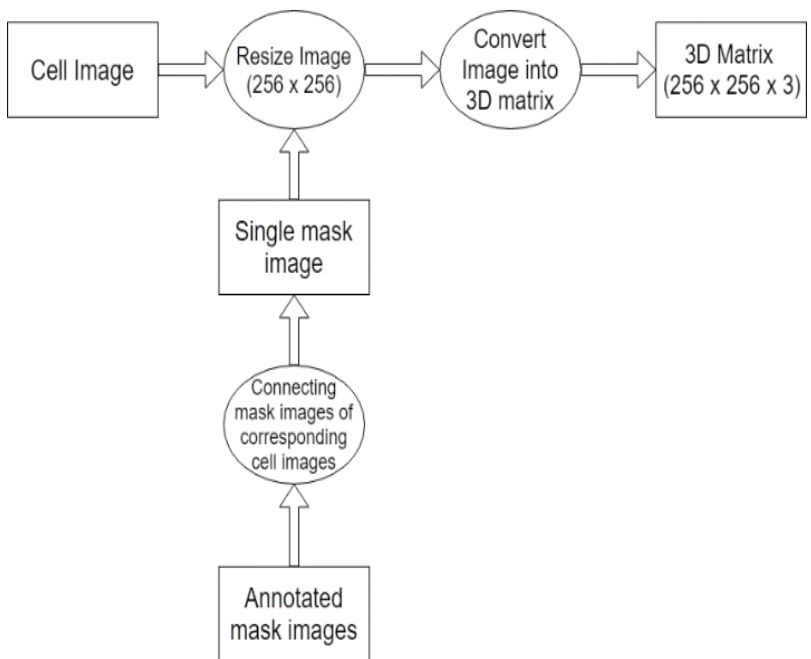

Fig. 3. Preprocessing module

Step 1. As mentioned in the above Data set sub-section (A), there are more than one mask image for each cell image.
Hence, all the mask images are concatenated into one image to form a single mask image representing all the masks of the corresponding single cell image

Step 2. Once this is done, both the cell images and its corresponding mask image are resized into 256 x 256 size. This transformation is performed to keep things consistent before feeding it to the U-Net model

Step 3. After each image is converted into a 3D matrix of size $256 \times 256 \times 3$, where the height and width are 256 and the depth i.e. the input channels (RGB values) is 3

At the end of preprocessing stage, the systems have generated data that a computer machine can understand. It is important to note that the process does not convert the images into grayscale equivalents. This is because the model can now train on different colored images, have a variety of inputs as well as avoid overfitting.

\section{C.Data Augmentation}

As stated by Jason Wang et al in [14], there is large consent that successful training of deep networks requires many thousand annotated training samples. For this reason, the system uses a network and training strategy that strongly relies on data augmentation to use the available annotated samples more efficiently [14].

Data augmentation is essential to teach the network the desired invariance and robustness properties, when comparatively few training samples are available.

Following are some of the parameters that are changed during data augmentation

1. Horizontal/Vertical split

2. Zoom range

3. Shear distance

4. Rotation angle etc.

\section{D.Model Architecture}

The model architecture is illustrated in Fig 4 at the end of the paper. It consists of following parts

\section{Contracting path [15], [16], [17], [18]}

The contracting path makes up a typical convolutional neural network. It consists of four layers. Each layer has a convolutional layer with a $3 \times 3$ kernel size, a padding to retain the original image dimension each followed by a ReLU (rectified layer unit) layer and a $2 \times 2$ max pooling layer at the end with a stride operation of 2 .

After each down-sampling phase, the number of filters for the $3 \times 3$ are doubled. This helps the system to learn only valid learnable parameters.

\section{Bottleneck}

This is the fifth convolutional layer that comes under the left contracting part.

\section{Expanding path [12], [13], [20]}

This part is responsible for up-sampling the images to its original dimensions. Each layer in the expansive path consists of a $2 \times 2$ transposed convolution layer and a concatenation with the corresponding feature map at the

contracting path As stated in [21], the system uses cloud computing as a viable solution for processing loads of data with consideration for privacy

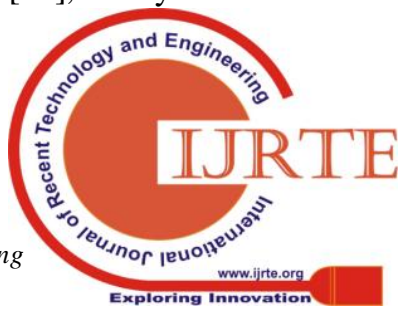


preservation.

The system uses GPU based training approach as GPUs are much faster than CPU in terms of graphical processing [22]. GPU can process multiple matrix multiplications simultaneously hence increases the training speed. The computing power thus increases multifold and training model is developed in a matter of minutes.

\section{RESULTS}

The research uses the following metrics for measuring the system's performance

\section{Accuracy [12], [13]:}

Accuracy is the ratio of number of correct predictions to the total number of predictions made. Higher the accuracy, better the classification

\section{Log loss (cost function) [12]:}

Accuracy quantification of a classifier is possible by $\log$ loss by penalizing false classifications. Maximizing the classifier accuracy is equivalent to minimizing of log loss.

\section{Mean Intersection over Union (IoU) [12]:}

For semantic image segmentation, a common evaluation metric is Mean Intersection-Over-Union where IOU for each semantic class is computed and then its results are averaged for all classes.

\section{A. Result Analysis}

Table III shows percentage values for the metrics mentioned above after every five epochs.

TABLE IV. Metric Analysis

\begin{tabular}{|l|c|c|c|}
\hline Epoch \# & Log loss & Accuracy & Mean IoU \\
\hline 1 & 68.00 & 84.31 & 44.11 \\
\hline 5 & -75.07 & 93.58 & 56.52 \\
\hline 15 & -79.01 & 95.69 & 64.20 \\
\hline 20 & -80.49 & 96.47 & 69.36 \\
\hline 25 & -81.04 & 96.89 & 72.48 \\
\hline 30 & -82.89 & 97.04 & 74.71 \\
\hline 35 & -83.58 & 96.98 & 76.22 \\
\hline 40 & -82.73 & 96.93 & 77.22 \\
\hline 45 & -81.09 & 96.39 & 78.11 \\
\hline 50 & -84.27 & 96.78 & 78.85 \\
\hline
\end{tabular}

\section{B. Visual Representation}

Fig 5, Fig 6, and Fig 7 display varied charts such as log loss curve, accuracy curve and mean IoU curve respectively for visual representation of the metric analysis.

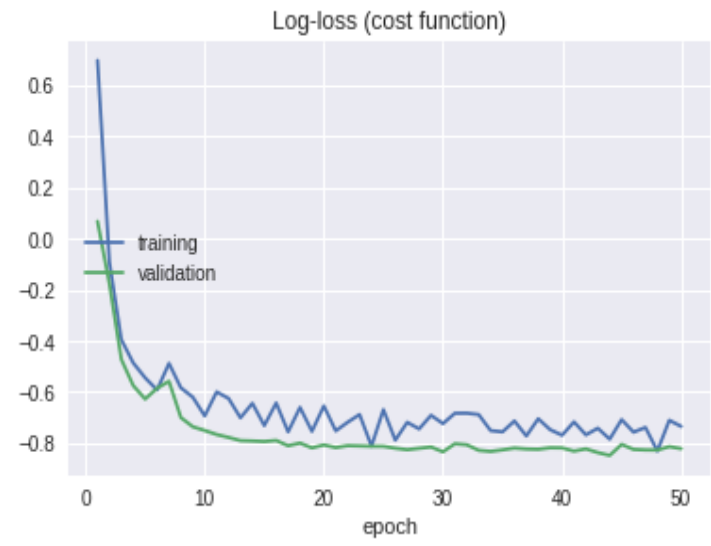

Fig. 5. Log loss curve

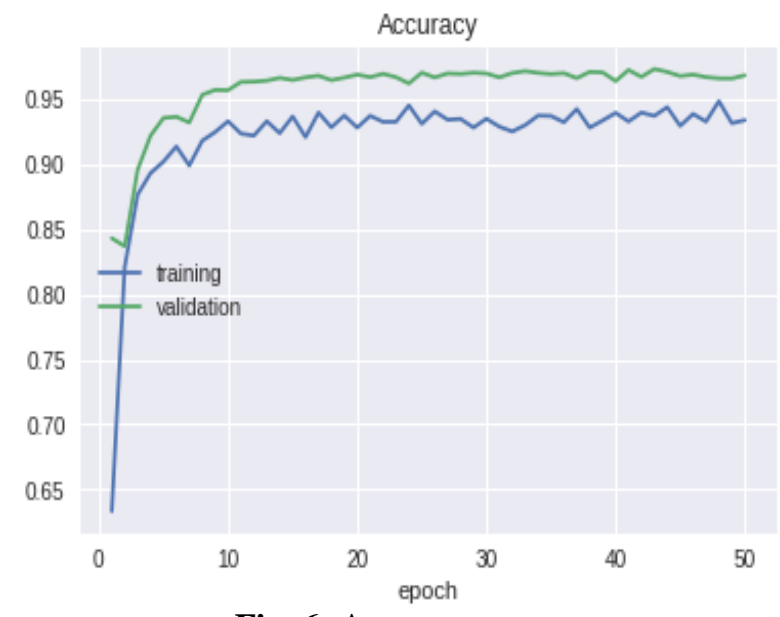

Fig. 6. Accuracy curve

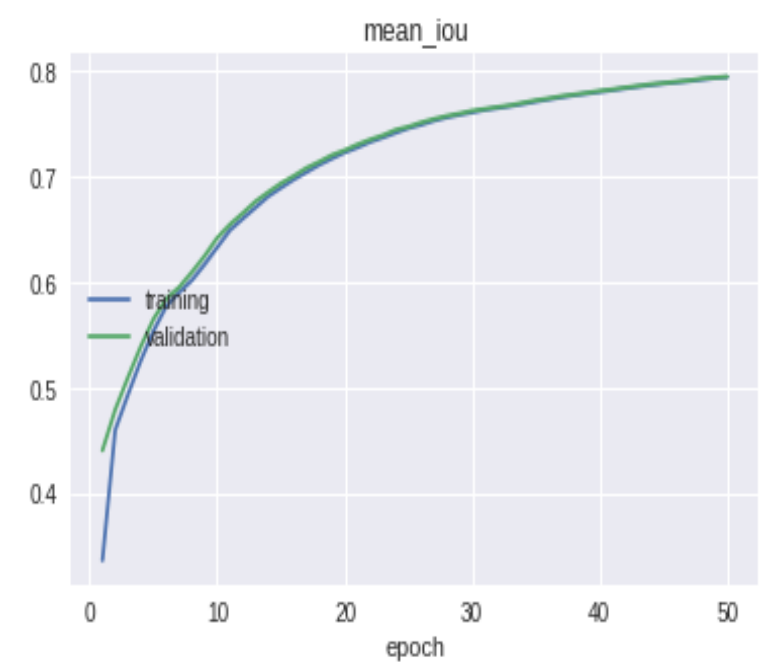

Fig. 7. Mean IoU curve

\section{Result Summary}

- It can be observed from Fig 5 that the loss decreases with each increasing epoch, hence model's failure rate decreases.

- From Fig 6, it can be observed that the accuracy increases with each increasing epoch

- In Fig 7, it can be observed that the mean intersection over union of the training set and the validation set almost overlaps with each other after few epochs. This shows that the original nuclei mask and the resultant nuclei mask falls in the same region.

\section{Result Evaluation}

Fig 8 shows the input cell 
image whose nuclei regions needs to be found. The resultant image is shown in Fig 9 where the system correctly spots the nuclei areas of the input cell image.

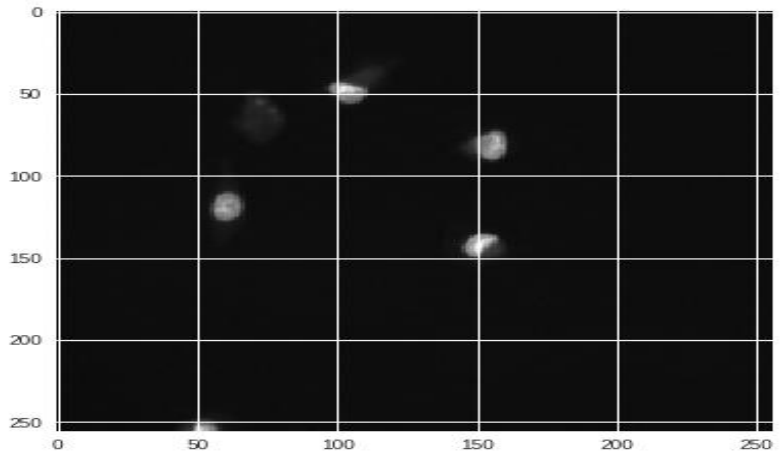

Fig. 8. Input cell image

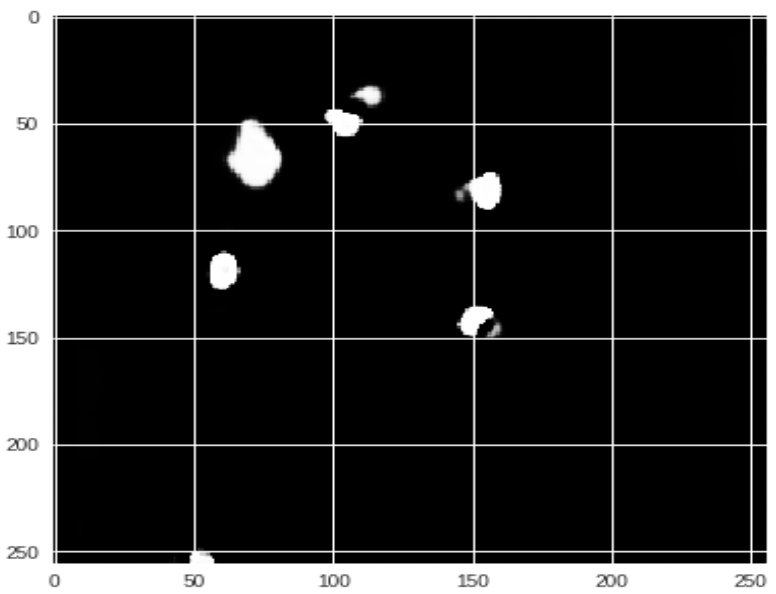

Fig. 9. Output image with spotted nuclei

The above evaluation depicts a very simple scenario where the nuclei are fairly round and not crowded with close neighboring nucleus and it also does not have any tissue or muscle areas. But as discussed in above sections, this model is able to spot nuclei even in images with crowded nucleus as seen in Fig 10 and Fig 11. In Fig 10, the input cell image has nuclei with close neighbors or with more tissue and muscle areas.
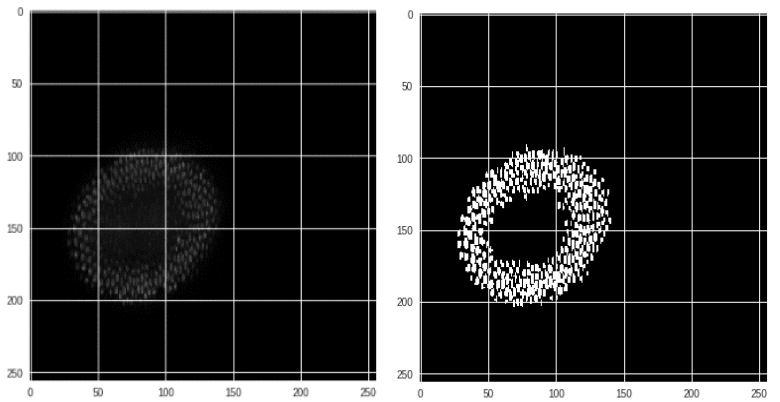

Fig. 10. Cell Image with crowded nucleus

It shows that even if the input cell image has several crowded neighboring nuclei the model produces significantly accurate results.
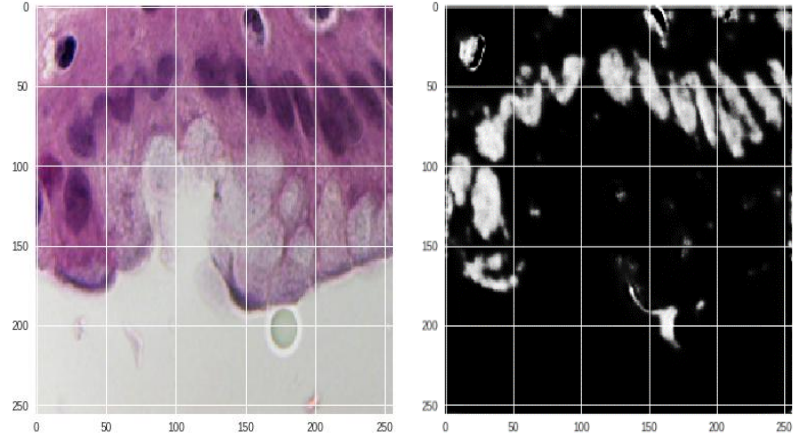

Fig. 11. Cell Image with tissue and muscle stain As illustrated in Fig 11, the model works accurately even if There are tissue or muscle areas along with nucleus. The model successfully removes muscle tissues and only spots nuclei region producing significant results.

\section{CONCLUSION}

This system through experimentation on medical cell images can predict nuclei position accurately using machine learning/ deep learning model. Biologist intervention in nuclei spotting can be completely eliminated. The use of U-Net framework for training the model and the GPU based solution improves the training performance significantly. The overall time required in drug discovery process considerably reduces due to the incorporation of this proposed system.

\section{FUTURE SCOPE}

The system can be further improved by predicting the impact of chemical that was tested on a particular batch of cell. Analysis of the overall change in the actual nuclei before testing and after the testing process can reveal significant insights by which the system can automatically deduce if a chemical was prone useful or harmful for that batch of cell. This can significantly fasten the overall process and the whole drug discovery process can be automated not just limited to nuclei spotting.

\section{REFERENCES}

1. Humayun Irshad, Student Member, IEEE, Antoine Veillard, Ludovic Roux, and Daniel Racoceanu, Member, IEEE, "Methods for Nuclei Detection, Segmentation, and Classification in Digital Histopathology: A Review-Current Status and Future Potential”, 2016

2. Rujuta, "Review of Nuclei Detection, Segmentation in Microscopic Images," 2017

3. Kaiming He, "Mask R-CNN", 2018

4. Ross Girshick, "Fast R-CNN" ,2015

5. Shaoqing Ren, "Faster R-CNN: Towards Real-Time Object Detection with Region Proposal Networks", 2016

6. Anurag Arnab, "Pixelwise Instance Segmentation with a Dynamically Instantiated Network", 2015

7. Tsung-Yi Lin, "Focal Loss for Dense Object Detection", 2016

8. Simon Jegou, "The One Hundred Layers Tiramisu: Fully Convolutional DenseNets for Semantic Segmentation", 2017

9. Assaf Arbelle, "Microscopy cell segmentation via adversarial neural networks', 2018

10. David Joon Ho, "Nuclei Segmentation of Fluorescence Microscopy Images Using Three Dimensional Convolutional Neural
Blue Eyes Intelligence Engineering \& Sciences Publication

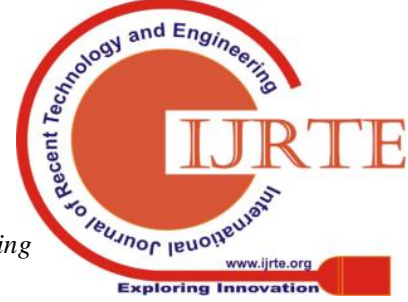


Networks", 2015

11. Tsung-Yi Lin, "Feature Pyramid Networks for Object Detection", 2016

12. Olaf Ronneberger, Philipp Fischer, and Thomas Brox, "U-Net: Convolutional Networks for Biomedical Image Segmentation", 2015

13. Rodney LaLonde, "Capsules for Object Detection", 2015

14. Jason Wang, Luis Perez, "The Effectiveness of Data Augmentation in Image Classification using Deep Learning", 2018

15. Tianmei Guo, Jiwen Dong ,Henjian Li'Yunxing Gao, "Simple Convolutional Neural Network on Image Classification", 2017

16. Ling Zhang, Milan Sonka, Le Lu, Ronald M. Summers, Jianhua Yao, "Combining fully convolutional networks and graph-based approach for automated segmentation of cervical cell nuclei”, 2017

17. Patrick Ferdinand Christ, "Automatic Liver and Tumor Segmentation of CT and MRI Volumes Using Cascaded Fully Convolutional Neural Networks", 2014

18. Yuxin Cui, "A Deep Learning Algorithm for One-step Contour Aware Nuclei Segmentation of Histopathological", 2018

19. Wei-Liang Tai, Chi-Shiang Chan, "Apply Run-length Encoding on Pixel Differences to Do Image Hiding", 2015

20. Sudhir Sornapudi, "Deep Learning Nuclei Detection in Digitized Histology Images by Superpixels", 2018

21. Deepali Vora, Deepali Nayak, "Privacy Preservation in SMAC-Social Networking, Mobile Network, Analytics and Cloud Computing", 2017

22. John D. Owens, Mike Houston, David Luebke, Simon Green, John E. Stone, and James C. Phillips, "GPU Computing", 2008

23. www.kaggle.com/gangadhar/nuclei-segmentation-in-mic roscope-cell-image 